University of Nebraska - Lincoln

DigitalCommons@University of Nebraska - Lincoln

USDA National Wildlife Research Center - Staff Publications
U.S. Department of Agriculture: Animal and Plant Health Inspection Service

2012

\title{
Yersinia pestis: examining wildlife plague surveillance in China and the USA
}

Sarah N. Bevins

US Department of Agriculture, Sarah.N.Bevins@aphis.usda.gov

John A. Baroch

USDA/APHIS/WS National Wildlife Research Center, john.a.baroch@aphis.usda.gov

Dale L. Nolte

USDA-APHIS-Wildlife Services, Dale.L.Nolte@aphis.usda.gov

Min Zhang

Chinese Academy of Sciences, Institute of Zoology, mzhang@msu.edu

Hongxuan $\mathrm{He}$

National Research Center for Wildlife Borne Diseases, hehx@ioz.ac.cn

Follow this and additional works at: https://digitalcommons.unl.edu/icwdm_usdanwrc

Bevins, Sarah N.; Baroch, John A.; Nolte, Dale L.; Zhang, Min; and He, Hongxuan, "Yersinia pestis: examining wildlife plague surveillance in China and the USA" (2012). USDA National Wildlife Research Center - Staff Publications. 1102.

https://digitalcommons.unl.edu/icwdm_usdanwrc/1102

This Article is brought to you for free and open access by the U.S. Department of Agriculture: Animal and Plant Health Inspection Service at DigitalCommons@University of Nebraska - Lincoln. It has been accepted for inclusion in USDA National Wildlife Research Center - Staff Publications by an authorized administrator of DigitalCommons@University of Nebraska - Lincoln. 


\title{
Yersinia pestis: examining wildlife plague surveillance in China and the USA
}

\author{
Sarah N. BEVINS, ${ }^{1}$ John A. BAROCH, ${ }^{1}$ Dale L. NOLTE, ${ }^{1}$ Min ZHANG ${ }^{2}$ and Hongxuan $\mathrm{HE}^{2}$ \\ ${ }^{1}$ US Department of Agriculture, Wildlife Services, National Wildlife Disease Program, Fort Collins, Colorado, USA and ${ }^{2}$ Chinese \\ Academy of Sciences, Institute of Zoology, Beijing, China
}

\begin{abstract}
Plague is a zoonotic disease caused by the bacterium Yersinia pestis Lehmann and Neumann, 1896. Although it is essentially a disease of rodents, plague can also be transmitted to people. Historically, plague has caused massive morbidity and mortality events in human populations, and has recently been classified as a reemerging disease in many parts of the world. This public health threat has led many countries to set up wild and domestic animal surveillance programs in an attempt to monitor plague activity that could potentially spill over into human populations. Both China and the USA have plague surveillance programs in place, but the disease dynamics differ in each country. We present data on plague seroprevalence in wildlife and review different approaches for plague surveillance in the 2 countries. The need to better comprehend plague dynamics, combined with the fact that there are still several thousand human plague cases per year, make well-designed wildlife surveillance programs a critical part of both understanding plague risks to humans and preventing disease outbreaks in the future.
\end{abstract}

Key words: plague, sentinel species, surveillance, Yersinia pestis

\section{INTRODUCTION}

Yersinia pestis is a Gram negative flea-borne bacterium and the causative agent of plague. The pathogen is traditionally described as cycling between small mammals, with an enzootic cycle and an epizootic cycle (Fig. 1). The enzootic cycle of plague is maintained among rodent hosts and their fleas; however, transmis-

Correspondence: Sarah Bevins, USDA National Wildlife

Disease Program, 4101 LaPorte Ave, Fort Collins, CO 80521, USA.

Email: Sarah.N.Bevins@aphis.usda.gov sion to humans and other mammals can occur (often during epizootic conditions) through flea bite or direct contact and, in some cases, results in severe morbidity and death (Barnes 1982; Gage \& Kosoy 2005). Y. pestis has been documented to infect more than 200 mammal species, but likely persists in only a small number of rodents that are relatively resistant to disease (Pollitzer 1960). It has also been suggested that plague continually cycles, even among susceptible species, but that the loss of a few rodents often goes unnoticed (Gage \& Kosoy 2005). These maintenance species differ depending upon the geographic location, or plague foci, in question. Plague foci are not static and foci presently exist in North America, South America, Africa and Asia. In Asia, some species of gerbil (i.e. Rhombomys opi- 


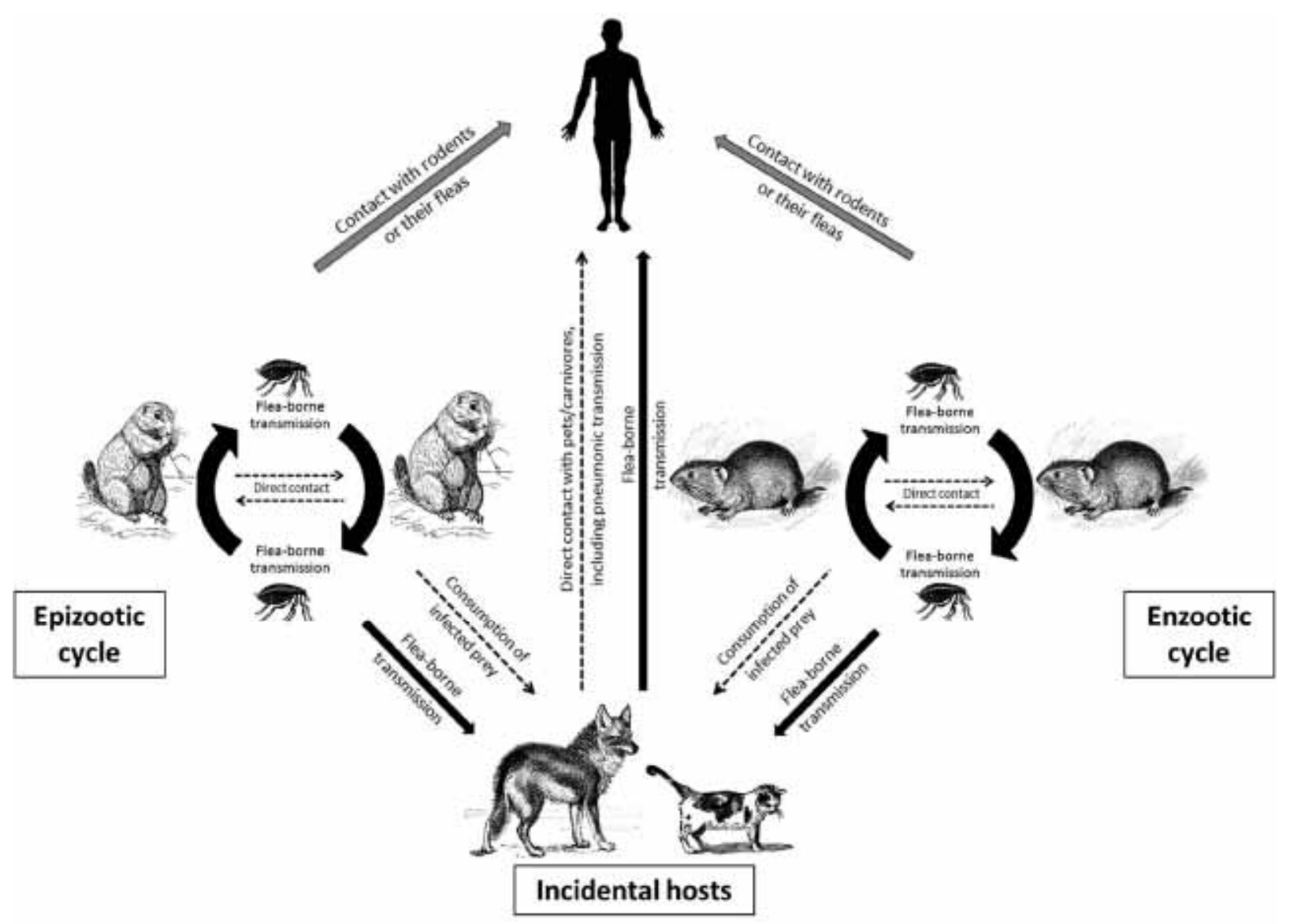

Figure 1 Basic plague transmission dynamics.

mus Wagner, 1841) and marmots (i.e. Marmota himalayana Hodgson, 1841) are resistant to infection and are thought to be maintenance hosts for $Y$. pestis (Pollitzer 1960), as are some small mammals in North America (i.e. Microtus californicus Peale, 1848) (Pollitzer 1960; Biggins \& Kosoy 2001). Carnivores and other species regularly exposed to plague likely mount an immune response that typically averts death, but these species are not believed to maintain the infection or to directly contribute to its transmission (Gage et al. 1994, 2000; Perry \& Fetherston 1997). Humans, along with felids, lagomorphs, prairie dogs and other rodents, are a group of mammals known to succumb to severe $Y$. pestis infections (Gasper 1997; Gage \& Kosoy 2005). The basic facets of plague (low-level cycling in rodents and their fleas with occasional epizootic events leading to morbidity and death in both wildlife and humans) are gener- ally well understood. The detailed dynamics that govern plague transmission, however, as well as the species involved in the USA, are still unknown, despite extensive research.

Plague manifests in symptomatic hosts as bubonic, pneumonic or septicemic, with untreated pneumonic and septicemic cases in humans being invariably fatal (Eisen et al. 2008). Bubonic plague is characterized by bacilli spreading to, and enlarging, lymph nodes (externally referred to as buboes) near the cutaneous site of a flea bite. In some cases, bubonic infections can progress to secondary septicemic disease, spreading to other organs via the bloodstream. Secondary septicemia can also follow a pneumonic infection originating from inhalation of infectious Y. pestis. From 1964 to 2003, the World Health Organization (WHO) documented 80744 
human plague cases around the world and more than 6500 deaths, but this is likely an underestimate (Dennis et al. 1999; WHO 2002, 2004). Plague is 1 of 6 original communicable diseases that member states are required to notify occurrence of to the WHO. It is considered a Category A infectious disease because of its ability to be transmitted via aerosol and because of the high death rates associated with airborne transmission. The ability to aerosolize also makes it a potential bioterrorism agent. These dangers, combined with continued plague presence punctuated by occasional outbreaks in countries across the globe, make plague surveillance an integral part of public health programs around the world.

\section{MONITORING AND SURVEILLANCE}

Comprehensive disease surveillance programs are essential to understanding the epidemiology of zoonotic diseases. The importance of a strong surveillance system is being increasingly recognized in an era where livestock, wildlife and people are capable of rapidly moving around the globe, increasing the opportunity for pathogen dispersal and novel disease introduction. Areas with comprehensive surveillance programs are better able to detect and respond to disease, rendering disease emergence less likely (Morner et al. 2002; Jones et al. 2008; Deliberto et al. 2009, 2011; Scotch et al. 2009; Rhyan \& Spraker 2010). Surveillance is essentially a system that can continuously collect and analyze information on both wild animal health and associated risk factors to control a disease in a population or community (Artois et al. 2006). A surveillance system implies a dedicated strategy of sample collection and analysis, followed by a pre-planned response when disease is detected or when it reaches a pre-determined level (i.e. proportion of collected samples that are seropositive). This is in contrast to monitoring, where the goal is simply to detect temporal trends, or changes in temporal trends, through the systematic recording of epidemiologic information (Deliberto et al. 2009, 2011). Monitoring does not include a response to mitigate or eradicate a disease. The WHO recommends that a successful plague surveillance program have 4 elements: human disease surveillance, investigation of epidemiology and epizootics, surveillance of known plague foci and long-term management of plague foci identified through surveillance efforts (Dennis et al. 1999; Scientific Committee on Vector-borne Diseases 2008).

A basic requirement for a successful public health disease response is accurate reporting and diagnosis of human disease occurrence. As a notifiable disease, a positive plague diagnosis is reported to the Centers for Disease Control (CDC) by State Departments of Health when they occur in the USA. In China, it is also mandatory that human plague cases be reported to the Department of Health or Centre for Health Protection (Scientific Committee on Vector-borne Diseases 2008). Plague also remains a WHO notifiable disease if the disease occurs outside of plague-endemic areas or is likely to initiate an epidemic spread. In the USA, plague is part of the National Notifiable Disease Surveillance System (NNDSS). This surveillance system encompasses animal plague surveillance (i.e. serosurveys of carnivores) and reports of human cases, as well as laboratory testing of fleas, animal tissues and serum specimens. Having a designated database for plague data, such as the NNDSS or the WHO Global Alert and Response system is crucial and provides continual data flow on human plague incidence, which enhances the probability of detecting an increase in disease and allows an early response to possible outbreaks. It should be noted however, that most of these systems are dedicated to human disease occurrence and any data collected from wild or domestic animals should be cataloged and analyzed as well.

The CDC and the WHO follow the same plague diagnosis protocol to ensure robust and accurate disease diagnosis. A confirmed plague diagnosis must meet at least 1 of the following conditions:

1. A clinical isolate is identified as $Y$. pestis through morphology and at least 2 of the following 4 diagnostics: phage lysis of the cultures at $20-25^{\circ} \mathrm{C}$ and $37^{\circ} \mathrm{C}$, F1 antigen detection, a confirmed $Y$. pestis biochemical profile or a positive polymerase chain reaction result.

2. Two serum specimens taken during early and late stages of the infection demonstrate a 4-fold anti-F1 antigen titer difference by agglutination testing.

3. A serum sample tested by agglutination has a titer of $>1: 128$ and the patient has no known previous plague exposure or vaccination history. All agglutination testing must include hemagglutination inhibition to demonstrate $Y$. pestis specificity (Dennis et al. 1999; WHO 2010).

In addition, several new, rapid plague detection assays have been developed and are being tested as quicker, less expensive alternatives to traditional plague diagnostics (Chanteau et al. 2003). In plague endemic regions, rapid lateral-flow immunochromatography tests can be used to confirm active plague infection (antigen) 
if no other option is available (WHO 2010). The WHO has published a recommended course of action and response to follow if positive human plague infections suggest an outbreak (WHO 2010).

Plague infections must be analyzed and diagnosed using these specific criteria to ensure consistent understanding of what constitutes a positive plague diagnosis. However, plague surveillance programs will not be consistent across countries or even geographic regions, and should be tailored according to the host and vectors involved, as well as to the local dynamics and transmission cycles. Plague is endemic in both China and the USA, but their respective programs demonstrate alternative surveillance approaches in each country.

\section{YERSINIA PESTIS SURVEILLANCE IN}

\section{THE USA}

In the USA, human plague infections are relatively rare; however, morbidity and death still occur when infections are misdiagnosed or are left untreated. The majority of human plague cases in the USA are associated with peridomestic transmission in non-urban areas, often involving bites from rodent fleas or even pneumonic transmission from contact with domestic pets. These dynamics are vastly different from the urban rat and flea transmission characteristic of the Black Death and other historic plague pandemics. From 1950 through 2009, 464 plague cases were reported in the USA (Brown et al. 2010). Despite this relatively limited occurrence in humans, evidence of plague exposure in regions of western USA in non-domestic rodents and carnivores is substantial.

Although tracking and monitoring human disease is vital, plague is essentially a disease of rodents and represents one of the many emerging zoonotic pathogens seen around the world (Stenseth et al. 2008; Butler 2009). Surveillance that extends to zoonotic reservoirs or sentinel animals could allow for early detection of increased disease activity prior to the onset of human disease. In the USA, human infections have previously been linked to epizootics, demonstrating the importance of animal-based surveillance (Barnes 1982; Brown et al. 2010). Even if the data are not used for predictive purposes, baseline zoonotic disease information can still reveal changes, such as range expansion or shifts in seasonality. In addition, understanding environmental conditions that lead to epizootic outbreaks is pivotal when it comes to preventing future disease outbreaks. Climate variables, such as time-lagged tempera- ture and seasonal precipitation, were found to be closely associated with the frequency of human plague cases in the USA (Enscore et al. 2002). Similarly, it has been suggested that long-term human plague occurrence in China might be related to variation in sea surface temperatures and oscillations (Zhang et al. 2007).

Plague is not endemic to the USA and, consequently, the disease dynamics differ when compared to other regions with a long evolutionary association with the pathogen. Ships leaving Hong Kong in the late 1800s during the Third Pandemic (roughly 1855-1950) carried plague to new regions of the world, including India and the USA, and by the time the pandemic was officially over, more than 12 million people had died in India and China alone (Morelli et al. 2010). Plague is believed to have been introduced to the USA with shipboard rats that escaped into Californian ports around 1900. It then began to spread eastward and has been documented in a majority of areas within the continental USA west of the 100th meridian, with regions of south-western USA having the highest levels of plague activity. There are no defined plague foci in the western USA like there are in China, even though some regions probably have different mammalian hosts and fleas involved in plague transmission. The lack of defined foci might be related to $Y$. pestis strains in the USA being much less diverse than those in China. The initial introduction event probably involved a limited number of strains (founder effect). With time, the bacterium will likely continue to adapt and diversify in relation to host, vector and environmental pressures (Zhou et al. 2004; Anisimov et al. 2004).

Like other areas in the world, plague activity in the USA is often difficult to detect for extended periods of time. There is typically limited evidence of plague transmission occurring on a daily basis. Rather, there are occasional epizootics that result in highly visible die-offs of some rodent species. Prairie dogs (Cynomys sp.) suffer the most dramatic die-offs in the USA, with up to 98\% dying in plague-affected populations (Biggins \& Kosoy 2001). Although documenting these die-offs is an efficient and low-cost way to monitor plague dynamics, they are often detected only after an epizootic has been underway and might not necessarily serve as an early warning system. Monitoring plague exposure, or seroprevalence, through active surveillance of other animals that can act as sentinel species is a viable option for monitoring plague dynamics.

In the USA, animal-based plague surveillance is carried out by multiple state health departments at the local level, as well as through research universities, the CDC 


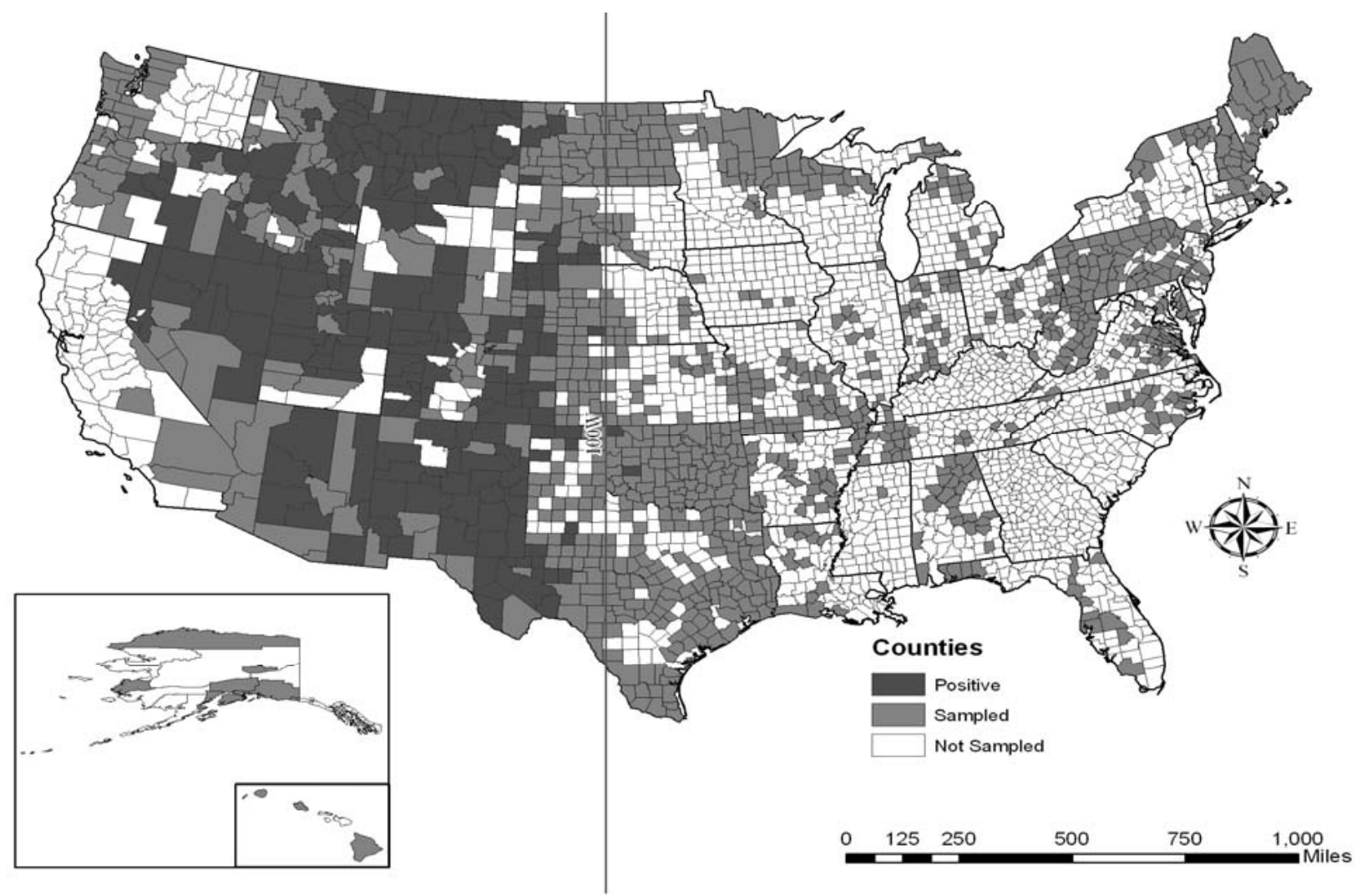

Figure 2 National Wildlife Disease Program (NWDP) sample sites in western USA from 2005-2009. Plague positive counties are indicated in black; the 100th meridian is marked with a grey line.

and the US Department of Agriculture (USDA). The USDA Animal and Plant Health Inspection Wildlife Services' National Wildlife Disease Program (NWDP) conducts continual plague surveillance in regions with documented plague activity in the USA, which is primarily restricted to areas west of the 100th meridian (Fig. 2). Plague surveillance by the NWDP is conducted through opportunistic sampling. Wildlife Services' specialists are requested to collect samples from select animals while conducting normal wildlife damage management operations in targeted areas. For example, blood samples from coyotes (Canis latrans Say, 1823) removed for preying on livestock are collected on Nobuto strips. Samples are collected by the NWDP and disseminated to the CDC for screening. Samples have been collected from multiple species since 2005. This information has helped to determine which species to target for collection over time and which species might serve as appropriate sentinels.
A sentinel species is essentially a group of animals that are sensitive to specific environmental conditions or abiotic factors and can therefore indicate changes in background conditions: in this case, disease activity. Numerous factors determine which species would work best as sentinels, including the target pathogen itself. For monitoring $Y$. pestis activity in the USA, the NWDP primarily focuses on coyotes. Coyotes are adaptable, wideranging, opportunistic omnivores found throughout much of western USA. They are ideal plague sentinels for several reasons (Gage et al. 1994; Aguirre 2009). Opportunistically sampling coyotes is efficient and cost effective when carried out in combination with already scheduled wildlife damage management activities. Coyotes have a broad diet and are known scavengers, essentially sampling from the environment while coming into contact with multiple rodent species and their fleas. Because they cover large distances, coyotes can act as disease bioaccumulators (dependent upon length of anti- 
body signal). Sampling a small number of wide-ranging carnivores provides a broader snapshot of disease activity when compared to sampling a small number of rodents. Equally important, when exposed to plague, coyotes appear to develop long-term antibodies (Rust et al. 1971; Barnes 1982) without morbidity or death.

Similar to coyote sampling in the USA, routine rabies vaccination campaigns in developing countries have opportunistically targeted domestic dogs (Canis lupus familiaris Linneaus, 1758) to screen for a variety of additional pathogens (Bogel \& Meslin 1990; Cleaveland et al. 2006). This approach might be a viable, cost-effective option for monitoring $Y$. pestis activity in countries where human plague outbreaks occur. Free-ranging domestic dog numbers are often high in urban areas in developing countries, with up to 1 dog for every 7-21 people (Cleaveland et al. 2006). Rabies vaccination along with a blood draw for disease screening would act as an incentive for dog owners. This approach was used successfully by Barnes (1982), and dogs showed increased seroprevalence prior to an epizootic and the associated human cases. Stray cats (Felis catus Linneaus, 1758) and dogs have been monitored for plague prevalence in Canada (Leighton et al. 2001). Sentinel animals are also used for a multitude of other pathogens, including monitoring rabbit hemorrhagic disease virus using foxes in New Zealand (Henning et al. 2006), sampling stray cats in Japan for q fever surveillance (Morita et al. 1994) and detecting bovine tuberculosis in the USA using coyote samples (VerCauteren et al. 2008).

Between 2005 and 2010, NWDP gathered and tested 25154 samples for plague from more than 70 species

Table 1 Sample sizes and average seropositivity for taxonomic family groups sampled from 2005-2009 in the USA

\begin{tabular}{lll}
\hline Species & Sample size & Plague seropositivity (\%) \\
\hline Canidae & 17882 & 10.20 \\
Felidae & 244 & 6.10 \\
Castoridae & 1298 & 0 \\
Sciuridae & 681 & 0.80 \\
Procyonidae & 929 & 0.60 \\
Muridae & 136 & 0 \\
Suidae & 348 & 3.70 \\
Mustilidae & 100 & 11 \\
Leporidae & 359 & 0.20 \\
\hline
\end{tabular}

collected across western USA (Fig. 2; Table 1). Of those samples, 21977 were from taxonomic groups that have been previously linked with $Y$. pestis (Table 1). One of the highest seroprevalence rates, averaged over 5 years of sampling, was seen in the Canidae, which includes samples from foxes, wolves and coyotes. Coyote accounted for $98 \%$ of those samples. Other carnivore species also displaying high $Y$. pestis seroprevalence included Mustelidae (badgers) and Felidae (pumas, bobcats, and domestic cats). Rodents are known to be integral players in plague transmission, but Sciuridae (squirrel species) and other rodent groups sampled have not revealed substantial plague exposure. This result is likely related to the difficulty of using continual, ongoing surveillance across a large geographic area to detect plague exposure in rodents. Rodent movements occur on a limited spatial scale and their population numbers are large, making it difficult to representatively sample across space. Targeted rodent sampling, however, is likely to yield useful results. For example, sampling around a house where an individual was exposed to plague or in an urban area where plague is known to occur. Using indices to calculate rat density or flea density as an indicator of plague activity could potentially be useful in these situations. The California Department of Public Health routinely monitors wild rodents in and around public campgrounds for plague antibodies and to assess flea loads of these species. High titers to Y. pestis or a high flea index will trigger flea suppression followed by rodent control measures (Anon. 2010). Although broadscale seroprevalence data provides valuable information on infectious disease dynamics, transmission and, therefore, seroprevalence, disease distribution will not be consistent across an entire region. Finer scale resolution would likely reveal disease hotspots, interspersed with areas/species with little to no disease activity.

\section{PLAGUE SURVEILLANCE IN CHINA}

Yersinia pestis evolved in or near China and later spread to other parts of the world through multiple radiation events (Zhou et al. 2004; Zhang et al. 2009; Morelli et al. 2010). The long-term association of $Y$. pestis in China has given rise to a diverse assemblage of plague transmission cycles and foci, a result of coupled evolution as the plague bacterium adapted to specific environmental factors, mammalian hosts and vector species. Within China, there are at least 10 natural plague foci (Table 2), each associated with specific host and flea species (Dennis et al. 1999). Recent genetic analy- 
Figure 3 Regions where wildlife was sampled for plague presence in China, from 2001-2008. Plague positive counties are indicated in black (Fu et al. 2009).

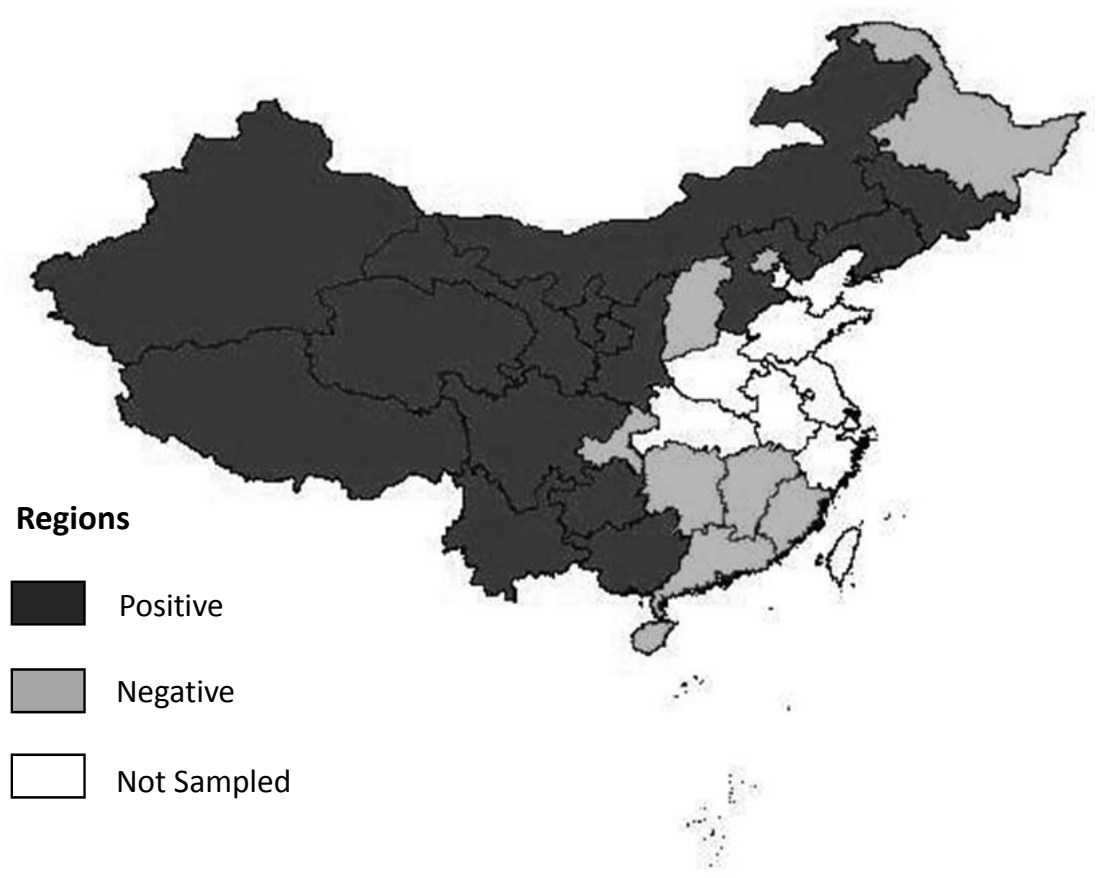

ses have indicated that there are 11-16 potentially separate foci (Ji et al. 1983; Zhou et al. 2004; Zhang et al. 2009).

Although there are multiple plague foci in China (Fig. 3), only a few are regularly associated with human plague cases. The foci linked to Marmota himalayan, the Himalayan marmot, is especially active (Table 2). This focus (which includes parts of Qinghai province and Tibet autonomous region) is thought to have continual, stable cycling of enzootic plague. Human exposure is typically associated with rural locations and either hunting, skinning or eating marmots. Interestingly, serologic surveys of marmot hunters and their relatives that live in this focus revealed previous plague exposure in $21.7 \%$ of study participants (Li et al. 2005). This suggests that exposure to $Y$. pestis is not uncommon and can result in asymptomatic infections, although anecdotal reports have also noted that some marmot hunters take antibiotics prophylactically (Li et al. 2005). In 2009, a pneumonic plague outbreak occurred within this focus after a herdsman had contact with 2 of his dogs that died from what was eventually determined to be $Y$. pestis infection (Wang et al. 2011). The man later died and 11 people who were known to have had direct contact with the man later contracted pneumonic plague as well, demonstrating the extremely infectious nature of the bacterium during pneumonic infections (Wang et al. 2011). Four marmots sampled from the area where the dogs were infected had substantial Y. pestis antibody titers. Domestic dogs are thought to be relatively resistant to plague infection; however, this case demonstrated that the conventional wisdom might not be universally applicable. Human plague infections, in both China and the USA, can often be traced back to contact with wild or domestic animals, highlighting the zoonotic nature of this disease and the need for robust plague surveillance in wildlife.

Another focus associated with human plague cases is in southern Yunnan Province, where the commensal rat, Rattus flavipectus Milne Edwards, 1872, is common. Whereas the previous focus was associated with marmots in rural landscapes, the focus linked to R. flavipectus often includes villages or urbanized areas and the commensal rats associated with them. After a 20 year period with no human plague cases reported from this focus, cases began occurring again in 1986. This focus now accounts for $50 \%$ or more of all human plague cases in China (Scientific Committee on Vector-borne Diseases 2008). Commensal rats are common in villages or cities, especially in areas where sanitation services are 


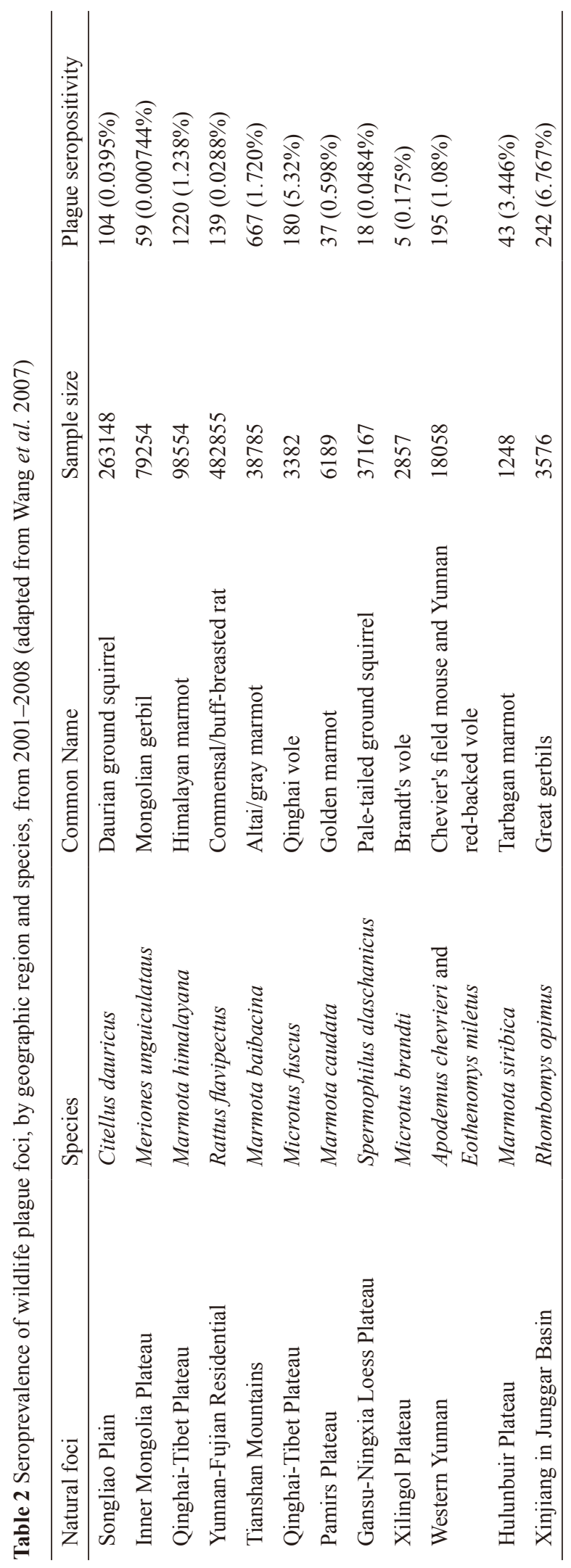

limited. Rattus flavipectus also often carries the flea species Xenopsylla cheopis Rothschild, 1903. This flea is considered the classic plague vector, abandoning its traditional rat host when the rat dies and taking up residence on other nearby mammal species, including dogs, cats and people. Xenopsylla cheopis is an efficient carrier/transmitter of the plague bacterium and the combination of both a suitable flea vector and a rat that lives in close association with people likely drives plague dynamics in Yunnan Province (Song et al. 2008).

The complexity of having numerous plague foci within a country requires that disease surveillance programs encompass both rural and urban transmission. A successful surveillance program must also incorporate multiple hosts and vector species, because each plague focus can involve different rodents and fleas. The vast size of the M. himalayana focus makes continual, routine plague surveillance difficult. One strategy that is used to reduce transmission is educating the local population about the risk of $Y$. pestis exposure when they are hunting or skinning marmots. During the 2009 pneumonic plague outbreak in Qinghai Province, the government responded quickly and quarantined an entire village of several thousand people to contain further transmission (Wang et al. 2011).

Surveillance programs in Yunnan Province and other foci associated with commensal rat species involve regular rodent trapping and flea collection. There are 2 factors that surveillance programs focus on to reduce rates of transmission in villages and urban areas. The first is to conduct regular surveillance of rodent densities. If measured densities surpass preset thresholds, control actions are triggered (e.g. rodent removal). Percent trap success (animals caught/number of traps set during a specific time period) is a commonly used index for assessing rodent density (Pham et al. 2009) and is often more feasible than using mark-recapture techniques to determine the absolute density of rodents per unit area (Dennis et al. 1999).

Although rodent control can help to limit plague hosts in and around domestic environments, any type of rodent control that is initiated must be accompanied by vector control. Fleas will often leave dying rodent hosts in search of another mammalian blood source, including humans and domestic animals. Rodent control without associated vector control may only increase $Y$. pestis transmission. Flea indices can also be calculated and a specific flea index can be used for each flea species of concern (number of a particular flea species collected from a particular host species/number of the particu- 
lar host species examined). Pollitzer (1954) notes that a specific flea index $>1$ for $X$. cheopis indicates a significant disease risk and control actions are recommended at this level. Application of insecticides for flea control can often significantly reduce plague risk, even in the absence of rodent control. Additional indices used in China to try and predict plague risk in urban areas are: the total flea index (total number of flea species collected regardless of species/total number of host species $\times$ that were examined); the percentage of hosts infected index (number of host species $\times$ that had at least 1 flea from a specific flea species/total number of host species $\times$ that were examined); and the house index (number of a specific flea species collected from host species $\times$ in a house/total number of houses with host species $\times$ examined). The house index can also be used for burrows or nests (Scientific Committee on Vector-Borne Disease 2008).

Flea indices are primarily used as a periodic sampling protocol to monitor the potential for plague activity in specific areas. Dramatic increases in flea indices over time might indicate the need for control measures. Flea indices are also used in both the USA and China, when there is a verified human plague case, but this falls outside of the realm of passive surveillance. Flea indices can be calculated, and fleas and blood samples from rodents can be tested for $Y$. pestis, to determine transmission dynamics and a likely route of exposure. However, flea indices can be unreliable and care must be taken in relation to the sampling technique and protocol, as well as data interpretation. All rodent trapping and flea collection should be standardized as much as possible to make valid temporal and spatial comparisons.

\section{CONCLUSION}

While human morbidity and death from plague has decreased since the advent of antibiotics, $Y$. pestis remains an endemic disease in many parts of the world. Infections in some regions are on the rise, to the point where plague is now classified as a reemerging disease (Jones et al. 2008; Stenseth et al. 2008). Misdiagnosis and delayed treatment can result in death, and plague can spread rapidly in rural or impoverished regions when action is not taken quickly or when medical help is not readily available.

Plague is a zoonotic disease and wildlife surveillance is crucial for not only pinpointing when epizootics occur, but also for the enhancement of a basic understanding of the pathogen. This concept is especially pertinent in the USA, where key rodent maintenance hosts remain unknown and where the recently introduced bacterium is still likely shifting in response to a variety of hosts and vectors. Wildlife surveillance in China has demonstrated that it is possible to identify increases in both fleas and mammalian hosts, allowing a rapid response to increased plague risk. While plague is endemic in both the USA and China, the 2 countries employ distinct surveillance strategies. $Y$. pestis has different evolutionary histories and ecological dynamics in each country. A successful surveillance strategy must take these potential variations into account and would ideally involve a one health approach (Enserink 2007), including biologists, epidemiologists, public health professionals, veterinarians and ecologists. Despite these differences, the end goal is the same: to respond quickly to disease outbreaks and, if possible, predict their occurrence and avoid human infections. Zoonoses represent a majority (60\%) of global emerging diseases (Jones et al. 2008) and are a risk not only to human populations, but also to domestic animals and wildlife, illustrating the continued importance of wildlife disease surveillance (Artois et al. 2006; Scotch et al. 2009).

\section{REFERENCES}

Aguirre AA (2009). Wild canids as sentinels of ecological health: a conservation medicine perspective. Parasites \& Vectors $\mathbf{2}, \mathrm{S} 7$.

Anisimov AP, Lindler LE, Pier GB (2004). Intraspecific diversity of Yersinia pestis. Clinical Microbiology Reviews 17, 434-64.

Anonymous (2010). California plague report, summer 2010. Vector-Borne Disease Section, Division of Communicable Disease Control, California Department of Public Health.

Artois M, Caron A, Leighton FA et al. (2006). Wildlife and emerging diseases. Revue Scientifique et Technique-Office International des Epizooties 25, 897912.

Barnes AM (1982). Surveillance and control of bubonic plague in the United States. 50, 237-70.

Biggins DE, Kosoy MY (2001). Influences of introduced plague on North American mammals: implications from ecology of plague in Asia. Journal of Mammology 82, 906-16.

Bogel K, Meslin FX (1990). Economics of human and canine rabies elimination: guidelines for programme orientation. Bulletin of the World Health Organization. 68, 281-91. 
Brown HE, Ettestad P, Reynolds PJ et al. (2010). Climatic predictors of the intra- and inter-annual distributions of plague cases in New Mexico based on 29 years of animal-based surveillance data. American Journal of Tropical Medicine and Hygiene 82, 95102.

Butler T (2009). Plague into the 21st Century. Clinical Infectious Diseases 49, 736-42.

Chanteau S, Rahalison L, Ralafiarisoa L et al. (2003). Development and testing of a rapid diagnostic test for bubonic and pneumonic plague. The Lancet 361, 211-6.

Cleaveland S, Meslin FX, Breiman R (2006). Dogs can play useful role as sentinel hosts for disease. Nature 440, 605.

Deliberto TJ, Swafford SR, Nolte DL et al. (2009). Surveillance for highly pathogenic avian influenza in wild birds in the USA. Integrative Zoology 4, 42639.

Deliberto TJ, Swafford SR, Nolte DL et al. (2011). Development of a national early detection system for highly pathogenic avian influenza in wild birds in the United States of American. In: Majumdar SK, Brenner FJ, Huffman JE et al., eds. Pandemic Influenza Viruses: Science, Surveillance, and Public health. Pennsylvania Academy of Sciences, Easton, pp. 156-75.

Dennis RT, Gage KL, Gratz N et al. (1999). Plague manual: epidemiology, distribution, surveillance and control. World Health Organization, Geneva.

Eisen RJ, Petersen JM, Higgins CL et al. (2008). Persistence of Yersinia pestis in soil under natural conditions. Emerging Infectious Diseases 14, 941-3.

Enscore RE, Biggerstaff BJ, Brown TL et al. (2002). Modeling relationships between climate and the frequency of human plague cases in the southwestern United States, 1960-1997. American Journal of Tropical Medicine and Hygiene 66, 186-96.

Enserink M (2007). Initiative aims to merge animal and human health science to benefit both. Science 316, 1553.

Fu QJ, Zhang CH, Cong XB (2009). Analysis results by etiology or serology for plague across China from 2001 to 2008. Chinese Journal of Control of Endemic Diseases 3, 187-9.

Gage KL, Kosoy MY (2005). Natural history of plague: perspectives from more than a century of research. Annual Review of Entomology 50, 505-28.
Gage KL, Thomas RE, Montenierti JA (1994). The role of predators in the ecology, epidemiology and surveillance of plague in the United States. Proceedings of the Sixteenth Vertebrate Pest Conference, California, Davis, 200-206.

Gage KL, Dennis DT, Orloski KA et al. (2000). Cases of cat-associated human plague in the Western US, 1977-1998. Clinical Infectious Diseases 30, 893900.

Gasper PW (1997). Plague. In: August JR, ed. Consultations in Feline Internal Medicine. W.B. Saunders, Philadelphia.

Henning J, Davies PR, Meers J (2006). Seropositivity to rabbit haemorrhagic disease virus in non-target mammals during periods of viral activity in a population of wild rabbits in New Zealand. Wildlife Research 33, 305-11.

Ji SL, Zhang HB, Liu YP (1983). The pattern of Yersinia pestis and its ecology significance in China. Zhongguo Yixие Kexие Yuan Xие Bao, 1-8 (In Chinese).

Jones KE, Patel NG, Levy MA et al. (2008). Global trends in emerging infectious diseases. Nature 451, 990-93.

Leighton F, Artsob H, Chu M et al. (2001). A serological survey of rural dogs and cats on the southwestern Canadian prairie for zoonotic pathogens. Canadian Journal of Public Health 92, 67-71.

Li M, Song Y, Li B et al. (2005). Asymptomatic Yersinia pestis infection, China. Emerging Infectious Diseases 11, 1494-6.

Morelli G, Song Y, Mazzoni CJ et al. (2010). Yersinia pestis genome sequencing identifies patterns of global phylogenetic diversity. Nature Genetics 42, 114043.

Morita C, Katsuyama J, Yanase T et al. (1994). Seroepidemiological survey of Coxiella burnetii in domestic cats in Japan. Microbial Immunology 38, 1001-3.

Morner T, Obendorf DL, Artois M et al. (2002). Surveillance and monitoring of wildlife diseases. Revue Scientifique et Technique-Office International des Epizooties 21, 67-76.

Perry RD, Fetherston JD (1997). Yersinia pestis - etiologic agent of plague. Clinical Microbiology Review 10, 35-66.

Pham HV, Dang DT, Tran Minh NN et al. (2009). Correlates of environmental factors and human plague: an ecological study in Vietnam. International Journal of Epidemiology 38, 1634-41. 
Pollitzer R (1960). A review of recent literature on plague. Bulletin of the World Health Organization 23, 313-400.

Rhyan JC, Spraker TR (2010). Emergence of diseases from wildlife reservoirs. Veterinary Pathology Online 47, 34-9.

Rust JH, Miller BE, Bahmanya M et al. (1971). Role of domestic animals in epidemiology of plague 2. Antibody to Yersinia pestis in sera of dogs and cats. Journal of Infectious Diseases 124, 527-31.

Scientific Committee on Vector-borne Diseases (2008). Situation of plague and prevention strategies. Centre for Health Protection, Hong Kong.

Scotch M, Odofin L, Rabinowitz P (2009). Linkages between animal and human health sentinel data. $B M C$ Veterinary Research 5, 15.

Song ZZ, Xia LX, Liang Y et al. (2008). Confirmation and study of plague natural foci for Yulong county and Guchengqu in Yunnan province. Chinese Journal of Control of Endemic Diseases 23, 3-7.

Stenseth NC, Atshabar BB, Begon M et al. (2008). Plague: past, present, and future. PLoS Medicine 5, e3.

VerCauteren KC, Atwood TC, DeLiberto TJ et al. (2008). Surveillance of coyotes to detect bovine tuberculosis, Michigan. Emerging Infectious Diseases 14, 1862-9.

Wang H, Cui Y, Wang Z et al. (2011). A dog-associated primary pneumonic plague in Qinghai province, China. Clinical Infectious Diseases 52, 185-90.
Wang YS, Liu QY, Cong XB et al. (2007). Plague reservoirs and their classification in natural foci of China. Chinese Journal of Vector Biology and Control 2, $127-33$.

World Health Organization (2002). Human plague in 2000 and 2001. WHO Weekly Epidemiological Report 78, 129-36.

World Health Organization (2004). Human plague in 2002 and 2003. WHO Weekly Epidemiology Record 79, 301-8.

World Health Organization (2010). Operational guidelines on plague surveillance, diagnosis, prevention and control. WHO Regional Office for South-East Asia, New Dehli.

Zhang X, Hai R, Wei J et al. (2009). MLVA distribution characteristics of Yersinia pestis in China and the correlation analysis. BMC Microbiology 9, 205.

Zhang Z, Li Z, Tao Y et al. (2007). Relationship between increase rate of human plague in China and global climate index as revealed by cross-spectral and cross-wavelet analyses. Integrative Zoology $\mathbf{2}$, $144-53$.

Zhou D, Han Y, Song Y et al. (2004). DNA microarray analysis of genome dynamics in Yersinia pestis: insights into bacterial genome microevolution and niche adaptation. The Journal of Bacteriology 186, 5138-46. 